\title{
Review: newer and older antidepressants have similar efficacy and total discontinuation rates but different side effects
}

Mulrow CD, Williams JW Jr, Trivedi M, et al. Treatment of depression: newer pharmacotherapies. Rockville, MD: Agency for Health Care Policy and Research; February 1999. AHCPR publication no. 99-E014. http://www.ahcpr.gov/clinic/deprsumm.htm.

QUESTION: In patients with depressive disorders, do newer and older pharmacotherapies have similar efficacy and adverse effects?

\section{Data sources}

Studies were identified by searching the Cochrane Collaboration Depression, Anxiety, and Neurosis Controlled Trials Registry; scanning trial article references and 46 meta-analyses; and contacting experts.

\section{Study selection}

Studies were selected if they were randomised controlled trials (RCTs) with an active intervention of $\geq 6$ weeks; compared a "newer" antidepressant with another antidepressant, placebo, or psychosocial intervention in patients with depressive disorders; and assessed depression symptoms, functional status, or quality of life.

\section{Data extraction}

2 reviewers independently extracted data on participant and diagnostic descriptors, setting, intervention, study methods, adverse effects, and outcomes (response rate, dropouts, and dropouts because of adverse events).

\section{Main results}

315 studies met the selection criteria. Similar response and total discontinuation rates existed for newer and older antidepressants. Newer antidepressants led to greater response rates in patients with major depression $(51 \%$ v 32\%, weighted relative benefit increase [RBI] $60 \%, 95 \%$ CI $50 \%$ to $70 \%$ ); dysthymia ( $59 \%$ v $37 \%$, weighted RBI $70 \%$, CI $30 \%$ to $130 \%$ ); and recurrent depression ( $47 \%$ v 28\%, weighted RBI $50 \%$, CI $20 \%$ to $90 \%$ ) than did placebo. SSRIs and tricyclic antidepressants led to similar response rates (RBI 0\%, CI -10 to 10), but specific side effects differed. SSRIs led to more patients with diarrhoea absolute risk difference (RD) $9 \%$, CI $5 \%$ to $13 \%$, , headache (RD 3\%, CI $0.2 \%$ to $4 \%$ ), insomnia $\{$ RD $6 \%$, CI $3 \%$ to $8 \%\} *$, and nausea (RD $10 \%$, CI $6 \%$ to $11 \%$ ), whereas tricyclics resulted in more patients with blurred vision (RD 4\%, CI $1 \%$ to $5 \%$ ), constipation (RD 12\%, CI 7\% to 14\%), dizziness (RD 11\%, CI $6 \%$ to $13 \%$ ), dry mouth (RD $30 \%$, CI $23 \%$ to $33 \%$ ), tremors (RD $4 \%$, CI $1 \%$ to $5 \%$ ), and urinary disturbance (RD $5 \%$, CI $1 \%$ to $8 \%$ ). Insufficient evidence exists to establish whether newer antidepressants are effective for other depressive syndromes or to compare them with psychosocial therapy. Methodological weakness compromised evidence for the effectiveness of St John's wort. First generation tricyclic agents led to a greater adverse effect related dropout rate.

\section{Conclusions}

Newer antidepressants are efficacious in the treatment of major depression and dysthymia. Response and discontinuation rates are similar among newer and older antidepressants, but specific side effects and related dropout rates differ. It is not known whether antidepressants are more effective than herbal treatment or psychosocial interventions.

*Information provided by author.

\section{COMMENTARY}

The introduction of SSRIs and other new antidepressants in the past decade increased the options available for tackling depressive illnesses. But has a major advance occurred in the treatment of this group of disorders so commonly encountered by family practitioners and hospital physicians? Mulrow et al's comprehensive report lends support to the findings of previous high quality systematic reviews that compared newer with older antidepressants. ${ }^{1}$ It also examines herbal remedies and psychosocial therapies.

All the meta-analyses have concluded that newer and older antidepressants have equivalent efficacy. Therefore, initial prescribing decisions have to be based on consideration of patient acceptability and cost. Dropout rates in RCTs have been used as the best available proxy measure for tolerability, and Mulrow et al found that tricyclic antidepressants and SSRIs are almost identical in this respect. However, side effect profiles differ, and patients should be informed of these differences so that their wishes can be considered when choosing medicine.

For complementary practitioners and their patients, some evidence exists that St John's wort has antidepressant properties, but more research is required.

Purchasers of health care should re-evaluate their spending on depression. The introduction of SSRIs has prompted a vast increase in spending, which is simply not justified by the available scientific data on efficacy and side effects.

This meticulously done meta-analysis highlights that the evidence does not address all common clinical problems. Insufficient research exists to guide prescribing in mild, severe, treatment resistant, or comorbid depressive illnesses. We cannot depend on the pharmaceutical industry to guide the use of important and widely used medicines; $94 \%$ of the antidepressant studies for which a funding source could be identified were industry financed. Health maintenance organisations, insurance companies, and especially governments should take note.

Anthony J Pelosi, MRCPsych Liz Ashton, MRCPsych Hairmyres Hospital Glasgow, UK

1 Geddes JR, Freemantle N, Mason J, et al. SSRIs versus other antidepressants for depressive disorder. Cochrane Review, latest version 22 Oct 1999. In: Cochrane Library. Oxford: Update Software. 